\title{
INTERACTIVE SMALL-STEP ALGORITHMS I: AXIOMATIZATION
}

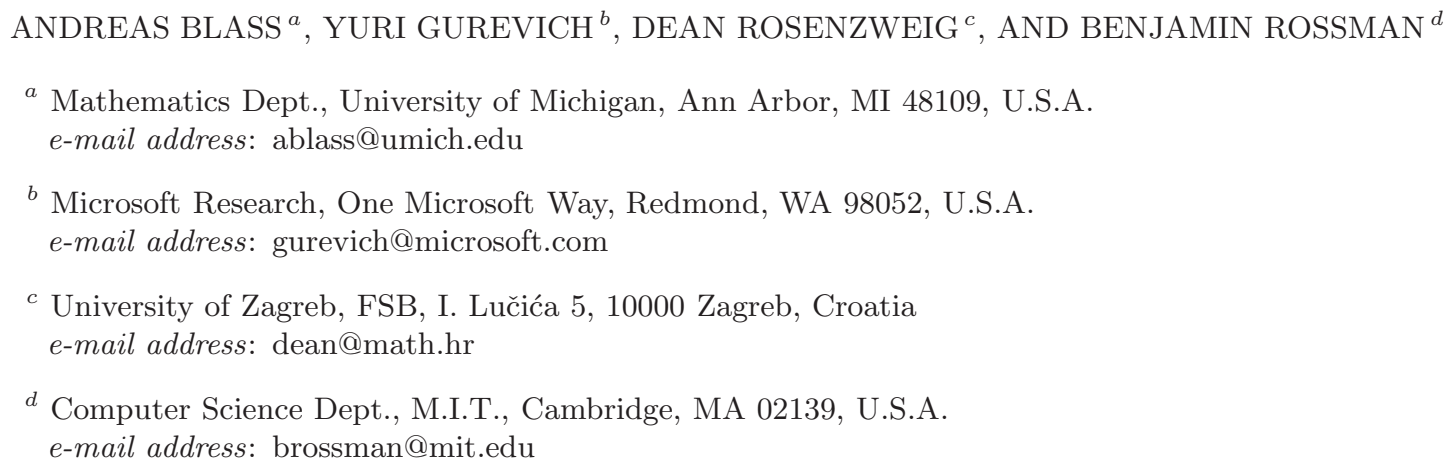

ABstract. In earlier work, the Abstract State Machine Thesis - that arbitrary algorithms are behaviorally equivalent to abstract state machines — was established for several classes of algorithms, including ordinary, interactive, small-step algorithms. This was accomplished on the basis of axiomatizations of these classes of algorithms. Here we extend the axiomatization and, in a companion paper, the proof, to cover interactive small-step algorithms that are not necessarily ordinary. This means that the algorithms (1) can complete a step without necessarily waiting for replies to all queries from that step and (2) can use not only the environment's replies but also the order in which the replies were received.

\section{INTRODUCTION}

The Abstract State Machine (ASM) Thesis, first proposed in 20] and elaborated in 21, 22, asserts that every algorithm is equivalent, on its natural level of abstraction, to an abstract state machine. Beginning in [23] and continuing in [5], 7], 8], and [9], the thesis has been proved for various classes of algorithms. In each case, the class of algorithms under consideration was defined by postulates describing, in very general terms, the nature of the algorithms. In each case the main theorem was that all algorithms of this class are

2000 ACM Subject Classification: F.1.1, F.1.2, F.3.1.

Key words and phrases: Interactive algorithm, small-step algorithm, abstract state machine, abstract state machine thesis, behavioral equivalence, ordinary algorithms, query.

${ }^{a}$ Blass was partially supported by NSF grant DMS-0070723 and by a grant from Microsoft Research.

${ }^{c}$ Rosenzweig was partially supported by the grant 0120048 from the Croatian Ministry of Science and Technology and by Microsoft Research.

-m 
equivalent, in a strong sense, to appropriate ASMs. The work in [23, 7, 8, 9] directly leading to the present paper is briefly overviewed in the following section.

The present paper continues this tradition, but with an important difference. Previously, the standard syntax of ASMs, as presented in [22, was adequate, with only very minor modifications. Our present work, however, requires a significant extension of that syntax. The extension allows an ASM program to refer to the order in which the values of external functions are received from the environment, and it allows the program to declare a step complete even if not all external function values have been determined.

The main purpose of this paper is to extend the analysis of interactive, small-step algorithms, begun in [7, 8, 9], by removing the restriction to "ordinary" algorithms. Here we provide postulates and definitions describing a general notion of interactive, small-step algorithm. In a companion paper [10] we also extend the syntax and semantics of abstract state machines (ASMs) so that non-ordinary algorithms become expressible. There we provide

- syntax and semantics for ASMs incorporating interaction that need not be ordinary,

- verification that ASMs satisfy the postulates, and

- proof that every algorithm satisfying the postulates is equivalent, in a strong sense, to an ASM.

The algorithms considered in this paper proceed in discrete steps and do only a bounded amount of work in each step ("small-step") but can interact with their environments during a step, by issuing queries and receiving replies.

Such algorithms were analyzed in [7, 8, 9], subject to two additional restrictions, which we expressed by the word "ordinary." First, they never complete a step until they have received replies to all the queries issued during that step. Second, the action of the algorithmthe queries issued and the next state produced - at any step is completely determined by the algorithm, the current state, and the function that maps the current step's queries to the environment's answers. In other words, this answer function is the only information from the environment that the algorithm uses. In particular, the order in which the environment's answers are received has no bearing on the computation.

In the present paper, we lift these restrictions. We allow an algorithm to complete a step even while some of its queries remain unanswered. We also allow the algorithm's actions to depend on the order in which answers were received from the environment. See the discussion in Section 3 and particularly Remark 3.5 for a discussion of why the order of arrival of answers is of special importance and why it cannot be subsumed in answer functions.

It was shown in 9] that ordinary algorithms are equivalent to ASMs of the traditional sort, essentially as described in 22]. In order to similarly characterize the more general algorithms of the present paper, we shall, in [10], extend the syntax and semantics of ASMs. In particular, we provide a way to refer to the timing of the evaluations of external functions and a way to terminate a step while some queries remain unanswered. See Subsection 3.2 for a discussion of the intuitive picture of interactive algorithms that leads to these particular extensions and indicates why they suffice.

At the referees' request, we comment briefly on the impact to be expected from this work. Numerous issues are involved here, which would be better addressed in a broad discussion of the ASM thesis rather than in this introduction, but it seems appropriate to say a few words about future prospects. Probably the most immediate impact that we 
expect from the ASM approach is on the foundations of software engineering. Clarification of commonly used notions - like state, level of abstraction, interaction - is long overdue. Closer to theoretical computer science, the ASM computation model is the most general computation model that we know. It supports for example computing with structures rather than strings; see [12] for particular examples leading to interesting theoretical results and questions. Finally it may be disappointing that, unlike the Church-Turing Thesis, the ASM thesis has not been used for negative results; will it be? It may. The attention of theorists is moving gradually toward the analysis of the overall behavior of algorithms and not only their input-output behavior.

\section{Related Work}

The previous work most closely related to the present paper is the series of papers [23, 5, 7, 8, 9], in which the ASM thesis is proved for other classes of algorithms - small-step algorithms in [23], parallel algorithms in [5] (both without external intra-step interaction, but with communication between subprocesses in the parallel case), and ordinary interactive small-step algorithms in [7, 8, 9]. Also, Andreas Glausch and Wolfgang Reisig [16, 17] adapted the postulates of [23] to describe a restricted but important class of "small-step" distributed algorithms. In this section, we briefly overview the work in [23, 7, 8, 9] directly leading to the present paper, and we also briefly discuss other work that is related to our goals and analysis, though it applies rather different methods.

2.1. Overview of the Behavioral Theory of Algorithms. Many algorithms are naturally understood on a high level of abstraction; for example states could be relational structures (databases). The step to a simulation operating over string representations of the abstract entities may lower the abstraction level of the algorithm; see section 2.2 for discussion. Such a step may be less trivial than meets the eye: for instance it is not known whether there is a polynomial-time algorithm that, given two adjacency matrices, determines whether they represent the same graph.

The Abstract State Machine (ASM) model of [22] intended to capture arbitrary algorithms at their natural levels of abstraction. The ASM Thesis of [22] asserts that every algorithm is equivalent, on its natural level of abstraction, to an abstract state machine. Subsequent experimentation provided confirmation of the thesis [2, 3, 13].

Paper [23] was the first of a series of papers offering speculative justification for the thesis, for particular classes of algorithms. They all follow the same general pattern;

(1) Describe axiomatically a class $\mathbf{A}$ of algorithms.

(2) Define behavioral equivalence of $\mathbf{A}$ algorithms.

(3) Define a class $\mathbf{M}$ of abstract state machines.

(4) Prove the following characterization theorem for $\mathbf{A}$ : $\mathbf{M} \subseteq \mathbf{A}$, and every $A \in \mathbf{A}$ is behaviorally equivalent to some $M \in \mathbf{M}$.

The characterization provides a theoretical programming language for $\mathbf{A}$ and opens the way for more practical languages for $\mathbf{A}$. The justification of the ASM Thesis thus obtained is speculative in two ways:

- The claim that $\mathbf{A}$ captures the intuitive class of intended algorithms is open to criticism.

- Definition of behavioral equivalence is open to criticism. 
But the characterization of $\mathbf{A}$ by $\mathbf{M}$ is precise, and in this sense the procedure proves the ASM thesis for the class of algorithms A modulo the chosen behavioral equivalence.

So A should be as broad as possible within the intended class of algorithms, and behavioral equivalence should be as fine as possible, even if coarser notions of equivalence might suffice for many purposes. The finer the behavioral equivalence, the stronger the characterization theorem.

In this subsection we briefly overview the realization of this program for isolated smallstep algorithms in [23] and ordinary interactive small-step algorithms in [7, 8, 9].

2.1.1. Isolated Small-Step Algorithms. The algorithms of [23] are executed by a single sequential agent and are isolated in the following sense: there is no interaction with the environment during the execution of a step. The environment can intervene in between algorithm's steps. But we concentrate on step-for-step simulation, and so inter-step interaction with the environment can be ignored. This class of algorithms is axiomatized by three simple postulates.

The Sequential Time Postulate says that an algorithm defines a deterministic transition system, a (not necessarily finite-state) automaton. More explicitly, the algorithm determines

- a nonempty collection of states,

- a nonempty subcollection of initial states, and

- a state-transition function.

The algorithm is presumed to be deterministic. Nondeterministic choices involve interaction with the environment; see [22, 23, 7] for discussion. The term state is used in a comprehensive way. For example, in case of a Turing machine, a state would include not only the control state but also the head position and the tape contents.

The postulate does not mention a notion of final state, see 23] for discussion.

Sequential Time already suffices to define behavioral equivalence of algorithms: two algorithms are behaviorally equivalent if they have the same

- states,

- initial states, and

- state-transition functions.

This is as fine as possible, though it may be too fine for many purposes. But, as already noted above, the finer the equivalence, the stronger the characterization theorem.

The Abstract State Postulate says that

- all states are first-order structures of a fixed vocabulary,

- the transition function does not change the base set of a state, and

- isomorphism of structures preserves everything, which here means states, initial states and the transition function.

It reflects the vast experience of mathematics and mathematical logic according to which every static mathematical situation can be adequately represented as a first-order structure. The idea behind the second requirement is that, even when the base set seems to increase with the creation of new objects, those objects can be regarded as having been already present in a "reserve" part of the state. What looks like creation is then regarded as taking an element from out of the reserve and into the active part of the state. (The nondeterministic choice of the element is made by the environment.) See [22, 23, 17, 8] and the next section for discussion. The idea behind the third requirement is that all 
relevant state information is reflected in the vocabulary: if your algorithm can distinguish red integers from green integers, then it is not just about integers.

The Bounded Exploration Postulate expresses the idea that a sequential algorithm (in the traditional meaning of the term) "computes in steps of bounded complexity" [26]. More explicitly, it asserts that the values of a finite set $W$ of terms (also called expressions), that depends only on the algorithm and not on the input or state, determine the state change (more exactly the set of location updates) for every step; see [22, 23] or the next section for precise definitions of locations and updates.

Thus, there is a uniform upper bound on the total size of locations that an algorithm needs to explore at any state. Bounded Exploration is the reason why all algorithms considered, here and in the rest of this paper, are called 'small-step'.

The characterization theorem of [23] establishes the ASM thesis for the class A of algorithms defined by Sequential Time, Abstract State, and Bounded Exploration and the class $\mathbf{M}$ of machines defined by the basic ASM language of update rules, parallel rules and conditional rules 21, 22, 23].

While the intent in [23] is to capture algorithms executing steps in isolation from the environment, a degree of intra-step interaction is accommodated in the ASM literature since [22]: (i) using the import command to create new elements, and (ii) marking certain functions as external and allowing the environment to provide the values of external functions. One pretends that the interaction is inter-step. This requires the environment to anticipate some actions of the algorithm. Also, in [22, nesting of external functions was prohibited; the first study of ASMs with nested external functions was [8]. The notion of run determines whether inter-step interaction is allowed, see [22, 23] for precise definitions and discussion.

2.1.2. Ordinary Interactive Small-Step Algorithms. In [7] it is argued at length why the inter-step form of interaction cannot suffice for all modeling needs. As a small example take the computation of $g(f(7))$ where $f(7)$ is an external call (a query) with argument 7 , whose result $a$ is used as the argument for a new query $g(a)$. An attempt to model this as inter-step interaction would force splitting the computation into substeps. But at some level of abstraction we may want to evaluate $g(f(7))$ within a single step. Limiting interaction to the inter-step mode would necessarily lower the abstraction level.

Thus [7] sets modeling intra-step interaction as its goal. Different forms of interaction, such as message-passing, database queries, remote procedure calls, inputs, outputs, signals, ... all reduce to a single universal form: a single-reply zero-or-more-arguments not-necessarily-blocking query. All arguments and the reply (if any) should be elements of the state if they are to make sense to the algorithm. For a formal definition of queries see [7]; a reminder is given in the next section. For a detailed discussion and arguments for the universality of the query-reply approach see [7].

Articles [7, 8, 9] limit themselves to interactive algorithms which are ordinary in the sense that they obey the following two restrictions:

(1) the actions of the algorithm depend only on the state and the replies to queries, and not on other aspects, such as relative timing of replies, and

(2) the algorithm cannot complete its step unless it has received replies to all queries issued. 
The first restriction means that an algorithm can be seen as operating on pairs of form $X, \alpha$ where $X$ is a state and $\alpha$ an answer function over $X$ : a partial function mapping queries over $X$ to their replies. The second restriction means that all queries issued are blocking; the algorithm cannot complete its step without a reply. (Some uses of nonblocking, asynchronous queries can still be modeled, by assuming that some forms of queries always obtain a default answer. But this is an assumption on environment behavior.) The present paper lifts both restrictions, and thus extends the theory to general interactive algorithms.

Several of the postulates of [7] cover the same ground as the postulates of [23], but of course taking answer functions into account. The most important new postulate is the Interaction Postulate, saying that the algorithm, for each state $X$, determines a causality relation $\vdash_{X}$ between finite answer functions and queries. The intuition behind $\alpha \vdash_{X} q$ is this: if, over state $X$, the environment behaves according to answer function $\alpha$ then the algorithm issues $q$. The causality relation is an abstract representation of potential interaction of the algorithm with the environment.

But not all answer functions can actually arise in such interaction. A context for $X, \vdash_{X}$ is a minimal (with respect to graph inclusion) answer function $\alpha$ with the following closure property: if $\beta \vdash_{X} q$ for some subfunction $\beta \subseteq \alpha$, then $q \in \operatorname{Dom}(\alpha)$. Intuitively, this means that $\alpha$ gives answers for exactly those queries that the algorithm would ask, given $\alpha$. This incorporates both the idea of blocking - all queries that are issued must be answered and the more basic idea that an algorithm cannot see replies to queries that it didn't issue. The Updates Postulate asserts that, for every context $\alpha$ for $X, \vdash_{X}$, the algorithm either fails or produces the next state.

This refines the transition relation of Sequential Time of [23]. The possibility of explicit failure is new here; the algorithm may obtain replies that are absurd or inconsistent from its point of view, and it can fail in such a case. The next state, if there is one, is defined by an update set, which can also contain trivial updates: "updating" a location to the old value. Trivial updates do not contribute to the next state, but in composition with other algorithms can contribute to a clash, see [7] and also the next section for discussion.

The inductive character of the context definition is unwound and analyzed in detail in [7. The answer functions which can occur as stages in the inductive construction of contexts are called well-founded. This captures the intuition of answer functions which can actually arise as records of interaction of an algorithm and its environment. Two causality relations (over the same state) are equivalent if they make the same answer functions well-founded. Equivalent causality relations have the same contexts but the converse is not in general true: intermediate intra-step behavior matters.

Two ordinary interactive algorithms are behaviorally equivalent if

- they have the same states and initial states,

- at all states they have equivalent causality relations, and

- at all state-context pairs, either they both fail or they produce the same update sets as required by Updates.

The Bounded Work Postulate of [7] extends Bounded Exploration of [23] to queries. As a consequence every well-founded answer function is finite. Furthermore, there is a uniform bound on the size of well-founded answer functions.

Queries can be expressed by external functions [22], but there is a complication. In some cases different textual occurrences of the same external function symbol applied to the same 
arguments can be reasonably assumed to define the same query, and thus to represent the same reply. In some cases, for instance with object-creation operators of OOP languages, different occurrences of the same external function symbol with the same arguments are always assumed to represent different queries which always obtain different fresh replies. There are also many intermediate possibilities. Most of [8] is devoted to a discussion of and a general solution to this problem using the device of query-templates, which we reuse here. In [8] the syntax and semantics of ordinary interactive ASMs is defined. The syntax is essentially the same as the ASM language of [23] with the addition of external function symbols, a subset of the ASM language of [22]. What is new is that the restriction of 22], forbidding nesting of external function symbols, can now be safely lifted: the query semantics and the analysis of causality provided by [7] did not exist at the time [22] was written. Thus the semantics of [22] was constrained to inter-step interaction only.

The characterization theorem for the class $\mathbf{A}$ of algorithms defined in [7] and the class $\mathbf{M}$ of machines defined in [8] is proved in [9].

2.2. Other Related Work. Efforts to characterize algorithms mathematically began with the work of Alonzo Church [14] and Alan Turing [34] in the 1930's. Turing gave a careful analysis and a gradual simplification of what can happen during an algorithmic computation performed by a person. The result was the Turing machine model of computation and a mathematical characterization, now universally accepted, of the functions (from finite strings to finite strings) that are computable in principle, disregarding limitations of time and memory. The simplifications, however, typically lower the level of abstraction in a drastic way; just compare any algorithm in [1] or [29] with a Turing machine implementation of that algorithm. The latter involves details - the data representation on the tape, indications how the read/write head should find the right place for the next "real" work, etc. - that are not essential to the algorithm (but essential for the Turing machine). Thus, although Turing's work successfully characterized the (in principle) computable functions, it characterized the algorithms that compute them only to the extent that one ignores levels of abstraction. ("In principle" means that there are no bounds on resources, so "computable" does not mean that we are able carry out the computation in the real world.)

The ASM thesis, in contrast, asserts that algorithms can be described at their natural levels of abstraction by ASMs. The textbook algorithms can be written as ASMs without introducing irrelevant details of implementation. A detailed implementation, such as a Turing machine or an assembly language program, can also be written as an ASM.

There are some other differences between the Turing machine model and the ASM model related to lowering of the abstraction level in the Turing machine model. In the Turing model, inputs and outputs are strings. ASMs deal naturally with computations involving abstract entities. Examples include the Gauss elimination algorithm over arbitrary fields and the ruler-and-compass constructions of geometry. Particularly important examples include computations with finite structures, e.g. relational databases. Databases can be implemented as strings. To do that, arbitrary choices may be necessary (to order the underlying sets). Thus string encoding exposes the implementation while database queries are supposed to be implementation independent. Furthermore, even when an algorithm works with strings, its parallel or interactive character can be lost in a Turing machine simulation.

Slightly before Turing's paper [34, Church [14 proposed another mathematical characterization of computability, in terms of definability in lambda-calculus. Church's proposal 
turned out to be equivalent to Turing's; that is, the lambda-definable functions are just those that are computable by Turing machines. Unlike Turing's proposal, however, Church's was not accompanied by an attempt to analyze the general notion of algorithm; it therefore lies considerably farther from our work.

Andrei N. Kolmogorov [26] has given another mathematical description of computation, presumably motivated by the physics of computation rather than by an analysis of the actions of a human computer. For a detailed presentation of Kolmogorov's approach, see [27. Also see [6] and the references there for information about research on pointer machines. Like Turing's model, these computation models also lower the abstraction level of algorithms.

Yiannis Moschovakis [30] proposed that the informal notion of algorithm be identified with the formal notion of recursor. A recursor is a monotone operator over partial functions whose least fixed point includes (as one component) the function that the algorithm computes. The approach does not seem to scale to algorithms interacting with an unknown environment. See [6, Section 4.3] for a critique of Moschovakis's computation model.

An approach to interactive computing was pioneered by Peter Wegner and developed in particular in [18. The approach is based on special interactive variants of Turing machines called persistent Turing machines, in short PTMs. Interactive ASMs can step-for-step simulate PTMs. Goldin and Wegner assert that "any sequential interactive computation can be performed by a persistent Turing machine" [19]. But this is not so if one intends to preserve the abstraction level of the given interactive algorithm. In particular, PTMs cannot step-for step simulate interactive ASMs [24.

The topic of the present paper is intra-step interaction between a single sequential-time algorithm and its environment (that is, the rest of the world). We are not aware of any previous literature on intra-step interaction. On the other hand, the communication mechanism that emerges from our study is not limited to intra-step interaction. Our investigation did not happen in a vacuum. It is hard for us to indicate particular sources that influenced our thinking, but surely — consciously or unconsciously — our thinking was influenced by the theory of distributed algorithms, by programming languages attempting to provide means for communication between computing agents, and by computing technology. A few references that seem relevant to us are [4, 28, 31, 32, 33].

\section{Postulates for Algorithms}

This section is devoted to the description of interactive, small-step algorithms by means of suitable definitions and postulates. Some parts of this material are essentially the same as in [23], which dealt with small-step algorithms that interact with the environment only between steps; some other parts are as in [7, which dealt with small-step algorithms that interact with the environment within steps but only in the "ordinary" manner described in the introduction. We shall present these parts again here, without repeating the explanations and motivations from [23] and [7], but see section 2.1. For the genuinely new material, dealing with the non-ordinary aspects of our algorithms' interaction with the environment, we shall present not only the definitions and postulates but also the reasons and intuitions that lie behind them.

In the following presentation of the postulates and their intuitive motivation, we use the word "algorithm" (or "interactive, small-step algorithm") in an informal sense. Our discussion amounts to an analysis of this intuitive notion, and our postulates are a distillation 
of the results of the analysis. Afterward, in Definition 3.24, we give the phrase "interactive small-step algorithm" a formal meaning, based on the postulates. Thus, Definition 3.24 implicitly asserts a thesis, namely that the postulates and the formal notion defined from them adequately capture the intuitive notion that we analyzed. In [10], we shall show that algorithms in our formal sense are behaviorally equivalent to ASMs. In the light of this result, the thesis implicit in Definition 3.24 is really the ASM thesis for interactive small-step algorithms.

Throughout the definitions and postulates that follow, we consider a fixed algorithm $A$. We may occasionally refer to it explicitly, for example to say that something depends only on $A$, but usually we leave it implicit.

3.1. States and vocabularies. Our first postulate is a cosmetic improvement of the State Postulate of [7], and most of it is assembled from parts of postulates in [23]. We refer the reader to [23] for a careful discussion of the first three parts of the postulate and to [7] for the last part.

States Postulate: The algorithm determines

- a finite vocabulary $\Upsilon$,

- a nonempty set $\mathcal{S}$ of states which are all $\Upsilon$-structures,

- a nonempty subset $\mathcal{I} \subseteq \mathcal{S}$ of initial states,

- a finite set $\Lambda$ of labels (to be used in forming queries).

As in the cited earlier papers, we use the following conventions concerning vocabularies and structures.

\section{Convention 3.1.}

- A vocabulary $\Upsilon$ consists of function symbols with specified arities.

- Some of the symbols in $\Upsilon$ may be marked as static, and some may be marked as relational. Symbols not marked as static are called dynamic.

- Among the symbols in $\Upsilon$ are the logic names: nullary symbols true, false, and undef; unary Boole; binary equality; and the usual propositional connectives. All of these are static and all but undef are relational.

- An $\Upsilon$-structure consists of a nonempty base set and interpretations of all the function symbols as functions on that base set.

- In any $\Upsilon$-structure, the interpretations of true, false, and undef are distinct.

- In any $\Upsilon$-structure, the interpretations of relational symbols are functions whose values lie in $\left\{\right.$ true $\left._{X}, \mathrm{false}_{X}\right\}$.

- In any $\Upsilon$-structure $X$, the interpretation of Boole maps true $X$ and false $_{X}$ to true $_{X}$ and everything else to false . .

- In any $\Upsilon$-structure $X$, the interpretation of equality maps pairs of equal elements to true $X$ and all other pairs to false .

- In any $\Upsilon$-structure $X$, the propositional connectives are interpreted in the usual way when their arguments are in $\left\{\operatorname{true}_{X}, \mathrm{false}_{X}\right\}$, and they take the value $\mathrm{false}_{X}$ whenever any argument is not in $\left\{\operatorname{true}_{X}, \mathrm{false}_{X}\right\}$.

- We may use the same notation $X$ for a structure and its base set.

- We may omit subscripts $X$, for example from true and false when there is no danger of confusion. 
Definition 3.2. A potential query in state $X$ is a finite tuple of elements of $X \sqcup \Lambda$. A potential reply in $X$ is an element of $X$.

Here $X \sqcup \Lambda$ means the disjoint union of $X$ and $\Lambda$. So if they are not disjoint, then they are to be replaced by disjoint isomorphic copies. We shall usually not mention these isomorphisms; that is, we write as though $X$ and $\Lambda$ were disjoint.

3.2. Histories and interaction. Interaction of an algorithm with its environment has been analyzed at length in [7]. The analysis established that all forms of interaction reduce to queries and replies. The algorithms of [7, 8, 9] are insensitive to the relative timing of replies within a step, and the environment interaction during a step could be expressed there as an answer function mapping queries to their replies. The following simple example shows that the expressive power of answer functions is not sufficient in general.

Example 3.3. For a simple example, consider a broker who has a block of shares to sell. He asks two clients whether they want to buy the shares. Both of them reply that they want to buy the whole block. The broker will sell the shares to the client whose message reaches him first.

Definition 3.4. An answer function for a state $X$ is a partial map from potential queries to potential replies. A history for $X$ is a pair $\xi=\left\langle\dot{\xi}, \leq_{\xi}\right\rangle$ consisting of an answer function $\dot{\xi}$ together with a linear pre-order $\leq_{\xi}$ of its domain. By the domain of a history $\xi$, we mean the domain $\operatorname{Dom}(\dot{\xi})$ of its answer function component, which is also the field of its pre-order component.

Recall that a pre-order of a set $D$ is a reflexive, transitive, binary relation on $D$, and that it is said to be linear if, for all $x, y \in D, x \leq y$ or $y \leq x$. The equivalence relation defined by a pre-order is given by

$$
x \equiv y \Longleftrightarrow x \leq y \leq x .
$$

The equivalence classes are partially ordered by

$$
[x] \leq[y] \Longleftrightarrow x \leq y,
$$

and this partial order is linear if and only if the pre-order was.

The length of a linear pre-order is defined to be the order type of the induced linear ordering of equivalence classes. (We shall use this notion of length only in the case where the number of equivalence classes is finite, in which case this number serves as the length.)

We also write $x<y$ to mean $x \leq y$ and $y \not \leq x$. (Because a pre-order need not be antisymmetric, $x<y$ is in general a stronger statement than the conjunction of $x \leq y$ and $x \neq y$.) When, as in the definition above, a pre-order is written as $\leq_{\xi}$, we write the corresponding equivalence relation and strict order as $\equiv_{\xi}$ and $<_{\xi}$. The same applies to other subscripts and superscripts.

We use histories to express the information received by the algorithm from its environment during a step. The notion of answer function comes from [7, where these functions, telling which queries have received which replies, represented the whole influence of the environment on the algorithm's work. When algorithms are not required to be ordinary, then additional information from the environment, namely the relative order in which replies were received, becomes relevant to the computation. This information is represented by the pre-order part of a history. If $p, q \in \operatorname{Dom}(\dot{\xi})$ and $p<_{\xi} q$, this means that the answer $\dot{\xi}(p)$ 
to $p$ was received strictly before the answer $\dot{\xi}(q)$ to $q$. If $p \equiv_{\xi} q$, this means that the two answers were received simultaneously.

The rest of this subsection is devoted to explaining in more detail the intuition behind this formalization of the intuitive notion of the history of an algorithm's interaction with its environment during a step. We do not, however, repeat here Sections 2 and 4 of [7]. The first of these two sections explains in detail our reasons for using queries and replies as our model of the interaction between an algorithm and its environment. The second explains the reasons for our specific definitions of (potential) queries and replies. Here, we presuppose these explanations and adopt the query-reply paradigm of [7]; see also section [2.1. Our task now is to explain what is added to the picture from [7] when we remove the restriction to ordinary algorithms.

Much of the information provided by an environment can and should be viewed as being part of its replies to queries. This includes not only the information explicitly requested by the query but also such varied information as "how hard did the user (who is part of the environment) bang on the keyboard when typing this input" or "at what time was this input provided" if such information is relevant to the algorithm's execution. Thus, we can view such information as being included in the answer function $\dot{\xi}$, without referring to the second component of a history, the pre-order.

The purpose of the pre-order, on the other hand, is to represent the order in which the algorithm becomes aware of the environment's answers. Even if the environment provides a time-stamp as part of each reply, this order cannot be read off from the replies. The algorithm may become aware of the replies in an order different from that indicated by the time stamps. In the broker example 3.3, the broker will sell the shares to the client whose message reaches him first, even if the message from the other client is sent earlier.

Remark 3.5. Could the environment provide, as part of its replies, time stamps that tell when the algorithm becomes aware of the replies? If so, could we then proceed as in [7], where algorithms get information from the environment only via the answer function? In other words, could we dispense with the pre-order component in histories?

The idea of a time stamp telling not when the reply was sent but when it is received is not as absurd as it might appear. The factors affecting the time of receipt, such as the speed of connections over which the reply travels and the speed at which the algorithm is executed, are not specified by the algorithm and the state, so they are part of the environment, albeit a different part from that ordinarily involved in answering queries. So the complete environment could conceivably assemble replies that include the time that the algorithm sees them. Such an environment would be quite unusual, and of course it would have to be subject to the constraint that the time stamps accurately reflect at least the order in which replies are received. But, if such an environment can be imagined, could we use it, at least for theoretical purposes, to avoid the need for the pre-order compnent in histories? If so, then the present paper could stay closer to the work previously done in [7, 8, 9], and we would have been quite happy to avoid the extra work.

The pre-orders are, however, needed, even for theoretical purposes. Consider the broker example above. Part of the broker's algorithm tells him what to do if both clients reply. To model that in the style of [7], assuming that replies include accurate information about their arrival times, the algorithm would have to wait for both replies, read both time stamps, and act accordingly. But in fact, the algorithm does not wait for both replies. If it receives one reply, it acts on the basis of that reply without waiting for the other. 
In effect, this algorithm and others like it decide the relative order of receipt of two replies as soon as one of the replies is received. If one is received and the other isn't, then the received one is earlier. This common sense approach is unavailable if the timing comparisons have to be based on reading time stamps embedded in the replies. Thus, even if we constrained our environments to always provide time stamps that accurately indicate the time a reply is seen by the algorithm, we would still need machinery like that in the present paper to decide the relative order of two replies even if only one has been seen.

Remark 3.6. Even though the pre-order $\leq_{\xi}$ is about the replies, the formal definition says that it pre-orders the domain of the answer function $\dot{\xi}$, i.e., the set of queries. The reason is a technical one: Different queries may receive the same reply, and in that case the single reply could occur at several positions in the ordering. Each query, on the other hand, is issued just once because, in accordance with the conventions of [7, if what appears to be the same query is issued repeatedly, then we regard the repetitions as distinct queries (since they can receive different replies). Thus, the order in which replies are received can be conveniently represented by pre-ordering the associated queries. It may be more intuitive to think of pre-ordering the set of pairs (query, reply), i.e., the graph of the answer function. This view of the situation would make no essential difference, since these pairs are in canonical bijection with the queries.

We emphasize that the timing we are concerned with here is logical time, not physical time. That is, it is measured by the progress of the computation, not by an external clock. If external, physical, clock time is relevant, as in real-time algorithms, it would have to be provided separately by the environment, for it is not part of the program or of the state. The relevant values of the physical time could be regarded as the replies to repeated queries asking "what time is it?"

In particular, we regard a query as being issued by the algorithm as soon as the information causing that query (in the sense of the Interaction Postulate below) is available. This viewpoint would be incorrect in terms of physical time, for the algorithm may be slow to issue a query even after it has the prerequisite information. But we can regard a query as being logically available as soon as information causing it is present.

This is why we include, in histories, only the relative ordering of replies. The ordering of queries relative to replies or relative to each other is then determined. The logical time of a query is the same as the logical time of the last of the replies causing that query.

Our use of pre-orders rather than partial orders, i.e., our allowing two distinct replies to be received simultaneously, also reflects our concern with logical rather than physical time. One can argue that no two events are exactly simultaneous in the physical sense (though they could be so nearly simultaneous that one should treat them as simultaneous), but logical simultaneity is certainly possible. It means merely that whenever the computation had access to one of the replies it also had access to the other.

The linearity of the pre-ordering, i.e., the requirement that every two replies be received either in one order or in the other or simultaneously, formalizes the following important part of our view of sequential-time (as opposed to distributed) algorithms. (It is not a postulate of our formal development but an informal principle that underlies some of our definitions and postulates.) 
One Executor Principle A small-step algorithm is executed by a single, sequential entity. Even if there are (boundedly many) subprocesses running in parallel, they all operate under the control of a single, master executor.

The aspect of this principle that is relevant to the linearity of histories can be summarized in the following informal principle, in which the first part emphasizes the role of the master executor in the interaction with the environment, while the second part is a consequence of the sequentiality of the executor.

Holistic Principle The environment can send replies only to (the executor of) the algorithm, not directly to parts of it; similarly it receives queries only from the algorithm, not from parts of it. Any two replies are received by the algorithm either simultaneously or one after the other.

The pre-orders in our histories are intended to represent the order in which replies are received by the master executor. If there are subprocesses, then the master may pass the replies (or information derived from them) to these subprocesses at different times, but that timing is part of the algorithm's internal operation (possibly also influenced by the replies to other queries like "what time is it?"), not part of the environment interaction that histories are intended to model. The linearity of our pre-orders reflects the fact that they represent the ordering as seen by a single, sequential entity; this entity sees things in a sequence.

In more detail, we picture the execution of one step of an algorithm as follows. First, the algorithm (or, strictly speaking, its executor) computes as much as it can with only the information provided by the current state. This part of the computation, the first phase, will in general include issuing some queries. Then the algorithm pauses until it is "awakened" by the environment, which has replied to some (not necessarily all) of the queries from phase 1. The algorithm proceeds, in phase 2 , to compute as much as it can using the state, the new information from the environment, and the information recorded during phase 1 . Then it again pauses until the environment has provided some more replies (possibly to queries from phase 2 and possibly to previously unanswered queries from phase 1) and awakens the algorithm. Then phase 3 begins, and this pattern continues until the algorithm determines, in some phase, that it is ready to complete the current step, either by executing certain updates (computed during the various phases) of its state or by failing (in which case the whole computation fails and there is no next state).

The logical ordering of replies refers to the phases at which replies were received. That is, if $q_{1}<_{\xi} q_{2}$, this means that the reply $\dot{\xi}\left(q_{1}\right)$ to $q_{1}$ was received at an earlier phase than the reply $\dot{\xi}\left(q_{2}\right)$ to $q_{2}$. Similarly, $q_{1} \equiv_{\xi} q_{2}$ means that these two replies were received at the same phase.

Remark 3.7. We have assumed, in the preceding description of phases, that the algorithm is awakened to begin a new phase only when some new reply has been provided. One may worry that this is not general enough, that the environment can awaken the algorithm even when no new replies are available. The rest of this remark is devoted to discussing this issue and justifying our assumption; it can safely be skipped by readers not worried about such subtleties. In such a case, it is natural to assume that the algorithm, having determined that it has no new information with which to advance the computation, simply resumes its pause until awakened again. In order for the algorithm to be a small-step algorithm, such fruitless awakenings must happen only a bounded number of times per step, with the 
bound depending only on the algorithm. The reason is that, even if all the algorithm does when awakened is to observe that no new replies have arrived, this observing is work, and a small-step algorithm can do only a bounded amount of it per step.

It seems possible, however, for an algorithm to admit a few fruitless awakenings per step, and the results of the computation could even depend on these awakenings. Consider, for example, an algorithm that works as follows. It begins by issuing a query $q$ and pausing. When awakened, it outputs 1 and halts if there is a reply to $q$; otherwise, it pauses again. When awakened a second time, it outputs 2 if there is now a reply to $q$, and, whether or not there is a reply, it halts. Notice that, if $q$ receives the reply $r$, then whether this is seen at the first or the second awakening doesn't affect the history, which consists of the function $\{\langle q, r\rangle\}$ and the unique pre-order on its domain $\{q\}$. So the history fails to capture all the information from the environment that is relevant to the computation.

There are two ways to correct this discrepancy. One, which we shall adopt, is to regard the fruitless awakening as amounting to a reply to an implicit query, of the form "I'm willing to respond to awakening." (For several fruitless awakenings, there would have to be several such queries, distinguished perhaps by numerical labels.) Now the two scenarios considered above, where $q$ has received a reply at the first awakening or only at the second, are distinguished in the histories, because the reply to the new, implicit query will be simultaneous with the reply to $q$ in the one scenario and will strictly precede the reply to $q$ in the other scenario.

An alternative approach would be to avoid introducing such implicit queries but instead to replace the pre-order constituents of histories by slightly more complicated objects, "preorders with holes." The idea is that the scenario where an answer to $q$ is available only at the second awakening would be represented by the answer function $\{\langle q, r\rangle\}$ as above, but the pre-order would be replaced with something that says "first there's a hole and then q." Formalizing this is actually quite easy, as long as the pre-order is linear and the set $D$ to be pre-ordered is finite (as it will be in the situations of interest to us). A linear pre-order of $D$ with holes is just a function $p$ from $D$ into the natural numbers. For any $n$, the elements of $p^{-1}(\{n\})$ constitute the $n^{\text {th }}$ equivalence class, which may be empty in case of a hole. Ordinary pre-orders correspond to the special case where the image $p(D)$ is an initial segment of $\mathbb{N}$.

Although the approach using pre-orders with holes corresponds more directly to intuition, we prefer the first approach, with implicit queries, for two reasons. First, it allows us to use the standard terminology of pre-orders rather than introducing something new. Second, its chief disadvantage, the need for implicit queries, is mitigated by the fact that we need other sorts of implicit queries for other purposes. For example, in [7, Section 2], implicit queries modeled the algorithm's receptiveness to incoming (unsolicited) messages and to multiple answers to the same query. Notice that, in all cases, our implicit queries represent the algorithm's willingness to pay attention to something provided by the environment.

We next define initial segments of histories, which will serve to model the interaction with the environment part way through a step.

Definition 3.8. Let $\leq$ be a pre-order of a set $D$. An initial segment of $D$ with respect to $\leq$ is a subset $S$ of $D$ such that whenever $x \leq y$ and $y \in S$ then $x \in S$. An initial segment of $\leq$ is the restriction of $\leq$ to an initial segment of $D$ with respect to $\leq$. An initial segment of a history $\left\langle\dot{\xi}, \leq_{\xi}\right\rangle$ is a history $\left\langle\dot{\xi}\left\lceil S, \leq_{\xi}\lceil S\rangle\right.\right.$, where $S$ is an initial segment of $\operatorname{Dom}(\dot{\xi})$ with 
respect to $\leq_{\xi}$. (We use the standard notation $\uparrow$ for the restriction of a function or a relation to a set.) We write $\eta \unlhd \xi$ to mean that the history $\eta$ is an initial segment of the history $\xi$.

Notice that any initial segment with respect to a pre-order $\leq$ is closed under the associated equivalence $\equiv$. Notice also that if $\left\langle\dot{\eta}, \leq_{\eta}\right\rangle \unlhd\left\langle\dot{\xi}, \leq_{\xi}\right\rangle$ then $\leq_{\eta}$ is an initial segment of $\leq_{\xi}$. We also point out for future reference that, if two histories $\xi_{1}$ and $\xi_{2}$ are initial segments of the same $\xi$, then one of $\xi_{1}$ and $\xi_{2}$ is an initial segment of the other.

Intuitively, if $\left\langle\dot{\xi}, \leq_{\xi}\right\rangle$ is the history of an algorithm's interaction with the environment up to a certain phase, then a proper initial segment of this history describes the interaction up to some earlier phase.

Definition 3.9. If $\leq$ pre-orders the set $D$ and if $q \in D$, then we define two associated initial segments as follows.

$$
\begin{aligned}
& (\leq q)=\{d \in D: d \leq q\} \\
& (<q)=\{d \in D: d<q\} .
\end{aligned}
$$

The following postulate is almost the same as the postulate of the same name in [7]. The only difference is that we use histories instead of answer functions. This reflects the fact that the decision to issue a query can be influenced by the timing of the replies to previous queries.

Interaction Postulate For each state $X$, the algorithm determines a binary relation $\vdash_{X}$, called the causality relation, between finite histories and potential queries.

The intended meaning of $\xi \vdash_{X} q$ is that, if the algorithm's current state is $X$ and the history of its interaction so far (as seen by the algorithm during the current step) is $\xi$, then it will issue the query $q$ unless it has already done so. When we say that the history so far is $\xi$, we mean not only that the environment has given the replies indicated in $\dot{\xi}$ in the order given by $\leq_{\xi}$, but also that no other queries have been answered. Thus, although $\xi$ explicitly contains only positive information about the replies received so far, it also implicitly contains the negative information that there have been no other replies. Of course, if additional replies are received later, so that the new history has $\xi$ as a proper initial segment, then $q$ is still among the issued queries, because it was issued at the earlier time when the history was only $\xi$. This observation is formalized as follows.

Definition 3.10. For any state $X$ and history $\xi$, we define sets of queries

$$
\begin{aligned}
\operatorname{Issued}_{X}(\xi) & =\left\{q:(\exists \eta \unlhd \xi) \eta \vdash_{X} q\right\} \\
\operatorname{Pending}_{X}(\xi) & =\operatorname{Issued}_{X}(\xi)-\operatorname{Dom}(\dot{\xi}) .
\end{aligned}
$$

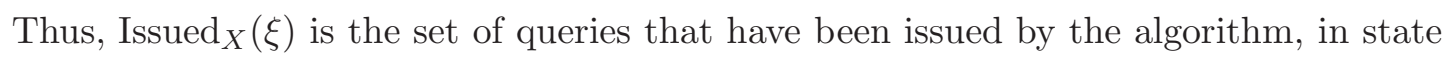
$X$, by the time the history is $\xi$, and $\operatorname{Pending}_{X}(\xi)$ is the subset of those that have, as yet, no replies.

Remark 3.11. We have described the causality relation in terms of detailed causes, histories $\xi$ that contain the algorithm's whole interaction with environment up to the time the caused query is issued. A text describing the algorithm, either informally or in some programming language, would be more likely to describe causes in terms of partial information about the history. For example, it might say "if the query $p$ has received the reply $a$ but $p^{\prime}$ has no reply yet, then issue $q$." This description would correspond to a large (possibly 
infinite) set of instances $\xi \vdash q$ of the causality relation, namely one instance for every history $\xi$ that fits the description, i.e., such that $\dot{\xi}(p)=a$ and $p^{\prime} \notin \operatorname{Dom}(\dot{\xi})$. More generally, whenever we are given a description of the conditions under which various queries are to be issued, we can similarly convert it into a causality relation; each of the conditions would be replaced by the set of finite histories that satisfy the condition.

The reverse transformation, from detailed causes representing the whole history to finite descriptions, is not possible in general. The difficulty is that the finite descriptions might have to specify all of the negative information implicit in a history, and this might be an infinite amount of information. In most situations, however, the set of queries that might be issued is finite and known. Then any finite history $\xi$ can be converted into a finite description, simply by adding, to the positive information explicit in $\xi$, the negative information that there have been no replies to any other queries among the finitely many that could be issued.

For semantical purposes, as in the present section, it seems natural to think of causes as being the detailed histories. From the behavior of an algorithm, one can easily determine whether $\xi \vdash_{X} q$ (at least when $\xi$ is attainable as in Definition 3.17 below and we ignore causes of $q$ that have proper initial segments already causing $q$ ); just provide the algorithm with replies according to $\xi$ and see whether it produces $q$ at that moment (and not earlier). It is not easy to determine, on the basis of the behavior, whether $q$ is issued whenever a description, like " $p$ has reply $a$ and $p^{\prime}$ has no reply" is satisfied, as this involves the behavior in a large, possibly infinite number of situations.

For syntactic purposes, on the other hand, descriptions seem more natural than detailed histories. And indeed, the syntax of our ASMs (in [10]) will not involve histories directly but will involve descriptions like " $p$ has reply $a$ and $p$ has no reply" in the guards of conditional rules.

Notice that there is no guarantee that $\operatorname{Dom}(\dot{\xi}) \subseteq \operatorname{Issued}_{X}(\xi)$, although this will be the case for attainable histories (defined below). In general, a history as defined above may contain answers for queries that it and its initial segments don't cause. It may also contain answers for queries that would be issued but only at a later phase than where the answers appear. In this sense, histories need not be possible records of actual interactions, between the algorithm and its environment, under the causality relation that is given by the algorithm. The following definition describes the histories that are consistent with the given causality relation. Informally, these are the histories where every query in the domain has a legitimate reason, under the causality relation, for being there.

Definition 3.12. A history $\xi$ is coherent, with respect to a state $X$ or its associated causality relation $\vdash_{X}$, if

(1) $(\forall q \in \operatorname{Dom}(\dot{\xi})) q \in \operatorname{Issued}_{X}(\xi\lceil(<q))$, and

(2) the linear order of the $\equiv_{\xi}$-equivalence classes induced by $\leq_{\xi}$ is a well-order.

The first requirement in this definition, which can be equivalently rewritten as

$$
(\forall q \in \operatorname{Dom}(\dot{\xi}))(\exists \eta \unlhd \xi)\left(\eta \vdash_{X} q \text { and } q \notin \operatorname{Dom}(\dot{\eta})\right),
$$

says that, if a query $q$ receives an answer at some phase, then it must have been issued on the basis of what happened in strictly earlier phases. In particular, the queries answered in the first phase, i.e., those in the first $\equiv_{\xi}$-class, must be caused by the empty history. 
The second requirement is needed only because we allow infinite histories; finite linear orders are automatically well-orders. The purpose of the second requirement is to support the usual understanding of "cause" by prohibiting an infinite regress of causes.

Remark 3.13. It will follow from the Bounded Work Postulate below in conjunction with the Interaction Postulate above, that we can confine attention to histories whose domains are finite. Then clause 2 in the definition of coherence would be automatically satisfied. Nevertheless, we allow a coherent history $\xi$ to have infinite domain and even to have infinitely many equivalence classes, with respect to $\leq_{\xi}$, provided their ordering induced by $\leq_{\xi}$ is a well-ordering. There are two reasons for this generality. First, it will allow us to state the Bounded Work Postulate in a relatively weak form and then to deduce the stronger fact that the histories we really need to consider (the attainable ones) are finite. Second, it avoids formally combining issues that are conceptually separate. Finiteness is, of course, an essential aspect of computation, especially of small-step computation, and the Bounded Work Postulate will formalize this aspect. But the notions of coherent and attainable histories are conceptually independent of finiteness, and so we do not impose finiteness in their definitions.

Remark 3.14. As mentioned above, the notion of coherence is intended to capture the idea of a history that makes sense, given the algorithm's causality relation. Here we implicitly use the fact that we are describing an entire algorithm, not a component that works with other components to constitute an algorithm. If we were dealing with a component $C$, working in the presence of other components, then it would be possible for queries to be answered without having been asked by $C$, simply because another component could have asked the query. See [11] for a study of components.

It follows immediately from the definitions that any initial segment of a coherent history is coherent.

Definition 3.15. A history $\xi$ for a state $X$ is complete if $\operatorname{Pending}_{X}(\xi)=\varnothing$.

Thus, completeness means that all queries that have been issued have also been answered. It can be regarded as a sort of converse to coherence, since the latter means that all queries that have been answered have also been issued earlier.

If a complete history has arisen in the course of a computation, then there will be no further interaction with the environment during this step. No further interaction can originate with the environment, because no queries remain to be answered. No further interaction can originate with the algorithm, since $\xi$ and its initial segments don't cause any further queries. So the algorithm must either proceed to the next step (by updating its state) or fail.

3.3. Completing a step. At the end of a step the algorithm will, unless it fails, perform some set of updates. The next postulate will express this property of algorithms formally. First, we adopt the formalization of the notion of update that was used in earlier work, starting with [23].

Definition 3.16. A location in a state $X$ is a pair $\langle f, \mathbf{a}\rangle$ where $f$ is a dynamic function symbol from $\Upsilon$ and $\mathbf{a}$ is a tuple of elements of $X$, of the right length to serve as an argument for the function $f_{X}$ interpreting the symbol $f$ in the state $X$. The value of this location in $X$ is $f_{X}(\mathbf{a})$. An update for $X$ is a pair $(l, b)$ consisting of a location $l$ and an element $b$ of $X$. 
An update $(l, b)$ is trivial (in $X$ ) if $b$ is the value of $l$ in $X$. We often omit parentheses and brackets, writing locations as $\left\langle f, a_{1}, \ldots, a_{n}\right\rangle$ instead of $\left\langle f,\left\langle a_{1}, \ldots, a_{n}\right\rangle\right\rangle$ and writing updates as $\langle f, \mathbf{a}, b\rangle$ or $\left\langle f, a_{1}, \ldots, a_{n}, b\right\rangle$ instead of $(\langle f, \mathbf{a}\rangle, b)$ or $\left(\left\langle f,\left\langle a_{1}, \ldots, a_{n}\right\rangle\right\rangle, b\right)$.

The intended meaning of an update $\langle f, \mathbf{a}, b\rangle$ is that the interpretation of $f$ is to be changed (if necessary, i.e., if the update is not trivial) so that its value at $\mathbf{a}$ is $b$.

Because our algorithms are not required to be ordinary, they may finish a step without reaching a complete history. The following postulate says that they must finish the step when they reach a complete history, if they have not already done so earlier.

Step Postulate - Part A The algorithm determines, for each state $X$, a set $\mathcal{F}_{X}$ of final histories. Every complete, coherent history has an initial segment (possibly the whole history) in $\mathcal{F}_{X}$.

Intuitively, a history is final for $X$ if, whenever it arises in the course of a computation in $X$, the algorithm completes its step, either by failing or by executing its updates and proceeding to the next step.

Since incoherent histories cannot arise under the algorithm's causality relation, it will not matter which such histories are final. We could have postulated that only coherent histories can be final, but there is no need to do so. It does no harm to allow incoherent final histories, though they are irrelevant to the algorithm's computation. By allowing them, we may slightly simplify the description, as algorithms subject to our postulates, of programs written in some programming language; if the language allows one to say "finish the step" then this can be directly translated into final histories, without having to check for coherence. Similar comments apply to the remaining parts of the Step Postulate below, where we require update sets or failure for coherent histories but allow them also for incoherent ones.

Definition 3.17. A history for a state $X$ is attainable (in $X$ ) if it is coherent and no proper initial segment of it is final.

Since initial segments of coherent histories are coherent, it follows that initial segments of attainable histories are attainable.

The attainable histories are those that can occur under the given causality relation and the given choice of final histories. That is, not only are the queries answered in an order consistent with $\vdash_{X}$ (coherence), but the history does not continue beyond where $\mathcal{F}_{X}$ says it should stop.

Our earlier statements to the effect that incoherent histories don't matter can be strengthened to say that unattainable histories don't matter. Thus, for example, although we allow one final history to be a proper initial segment of another (even when both are coherent), the longer of the two will be unattainable, so its being final is irrelevant.

Step Postulate - Part B For each state $X$, the algorithm determines that certain histories succeed and others fail. Every final, attainable history either succeeds or fails but not both.

Definition 3.18. We write $\mathcal{F}_{X}^{+}$for the set of successful final histories and $\mathcal{F}_{X}^{-}$for the set of failing final histories. 
Non-final or unattainable histories may succeed or fail or both, but this will be irrelevant to the behavior of the algorithm. If we restrict attention to attainable histories, then $\mathcal{F}_{X}^{+}$ and $\mathcal{F}_{X}^{-}$partition $\mathcal{F}_{X}$.

The intended meaning of "succeed" and "fail" is that a successful final history is one in which the algorithm finishes its step and performs a set of updates of its state, while a failing final history is one in which the algorithm cannot continue - the step ends, but there is no next state, not even a repetition of the current state. Such a situation can arise if the algorithm computes inconsistent updates. It can also arise if the environment gives inappropriate answers to some queries.

For more about failures, see the discussion following the Update Postulate in [7, Section 5]. There is, however, one difference between our present situation and that in [7]. There, the algorithm depended upon getting answers to all its queries, so it is reasonable to expect that an inappropriate answer always results in failure. In our present situation, however, the algorithm may be willing to finish its step without getting the answer to a certain query. In this case, an inappropriate answer to that query need not force failure.

When a history in $\mathcal{F}_{X}^{+}$arises during the computation, the algorithm should perform its updates and proceed to the next step. We formalize this in the third and last part of the Step Postulate, keeping in mind that only attainable histories can arise. The notation $\Delta^{+}$ for the update set is taken from [7. The superscript + refers to the fact that trivial updates can be included in the update set. Although they do not affect the next state, they can affect whether clashes occur when our algorithm is run in parallel with another 1 .

Step Postulate - Part C For each attainable history $\xi \in \mathcal{F}_{X}^{+}$for a state $X$, the algorithm determines an update set $\Delta^{+}(X, \xi)$, whose elements are updates for $X$. It also produces a next state $\tau(X, \xi)$, which

- has the same base set as $X$,

- has $f_{\tau(X, \xi)}(\mathbf{a})=b$ if $\langle f, \mathbf{a}, b\rangle \in \Delta^{+}(X, \xi)$, and

- otherwise interprets function symbols as in $X$.

The Step Postulate is the analog, for our present situation, of the Update Postulate of 7]. There are two differences between these postulates, reflecting the two requirements of ordinariness in [7] that have been lifted in the present work.

First, since we now consider algorithms that can finish a step without waiting for all queries to be answered, we include in the notion of algorithm the information about when to finish a step. This is the notion of final history introduced in Part A of the Step Postulate. If an algorithm never finishes a step while queries are still pending, then its final histories could be defined as being the complete histories (or the complete, coherent histories), and there would be no need to specify $\mathcal{F}$ separately in the algorithm. The complete, coherent histories correspond to the contexts, as defined in [7].

Second, because we allow an algorithm to take into account the order in which it receives replies to its queries, decisions about failures and updates depend not only on the answer function $\dot{\xi}$ but on the whole history $\xi$, including the ordering information contained in $\leq_{\xi}$.

The intuition and motivation behind the other aspects of the Step Postulate are discussed in detail in [23] and 7].

\footnotetext{
${ }^{1}$ In fact in this paper and its sequel [10, we do not define parallel composition of algorithms. But it is implicit in [9] and will be explicit in [11, and we wish to maintain uniformity of the framework across algorithm classes.
} 
Convention 3.19. In notations like $\mathcal{F}_{X}, \mathcal{F}_{X}^{+}, \mathcal{F}_{X}^{-}, \Delta^{+}(X, \xi)$, and $\tau(X, \xi)$, we may omit $X$ if only one $X$ is under discussion. We may also add the algorithm $A$ as a superscript if several algorithms are under discussion.

Notice that the Step Postulate requires $\Delta^{+}(X, \xi)$ and $\tau(X, \xi)$ to be defined when $\xi$ is an attainable, final, successful history, but it allows them to be defined also for other histories. Any values they may have for other histories, however, will not affect the algorithm's computation.

Notice also that the next state is completely determined by the current state and the update set. So when describing an algorithm, we need not explicitly describe $\tau$ if we have described $\Delta^{+}$.

If $\Delta^{+}(X, \xi)$ clashes, i.e., if it contains two distinct updates of the same location, then the description of $\tau(X, \xi)$ is contradictory, so the next state cannot exist. Thus, if such a $\xi$ is attainable and final, it must be failing. That is, clashes imply failure.

Example 3.20. We revisit the broker example 3.3 in terms of our definitions. Part of the broker's algorithm is easy to describe: the empty history causes two queries, $q_{0}(s, p, a)$ and $q_{1}(s, p, a)$. Query $q_{i}(s, p, a)$ expresses the take-it-or-leave-it offer to client $i$ to buy a block of $a$ shares of stock $s$ at price $p$. A history that contains a positive reply from just one client is final and successful and produces updates recording a sale to that client. Note that, if both clients reply positively but at different times, then the history as an initial segment containing only the reply from the client who answered first, so the stock is sold to this client. It is also easy to handle histories in which both clients answer negatively; such a history is successful and final but results in no updates (or perhaps in an update recording that these clients had the chance to buy the stock and turned it down). Note that, although this situation represents a failure of the broker to accomplish his aim, it should not be considered a failing history, as it does not mean that the broker goes out of business.

What if the broker receives positive replies from both clients at the same time? It is tempting and perhaps realistic to assume that this situation cannot occur, but that assumption amounts to a constraint on the environment, and we do not consider in this paper such a restriction of the theory to some "admissible" histories. Furthermore, when the broker really cannot receive two replies simultaneously, this will be because of some aspect of his receiving mechanism, and it seems that such aspects can be modeled using one of the following ideas.

The broker could systematically prefer one of the clients. Then a history with simultaneous positive replies from both clients would be final and successful and would produce updates recording a sale to the preferred client.

Alternatively, the broker could break ties non-deterministically. That is, a history with simultaneous positive replies from both clients would cause a new query to the environment, asking for a non-deterministic choice of a client. Any reply naming a client would result in a successful final history with a sale to that client.

To finish the specification of the algorithm, we must say what is to be done if netiher client replies, or if one replies negatively and the other doesn't reply. One approach is to declare such histories not to be final. The broker simply waits, perhaps forever.

A more realistic approach is that the broker's offers to the clients include a time limit. An easy way to model this is to have the empty history cause, in addition to the offers to the clients, a third query $t$, to be thought of as a time-out query, i.e., a request to be informed 
by the environment, when the time limit has been reached. Then any history with a reply to this query but no positive reply from a client will be final and successful and result in no update (or an update recording that there was no sale and why). The algorithm can still hang, if the environment fails to respond to the time-out query, but that seems a reasonable result for a broker who imposes a time-limit but can't find out when it has been reached.

Remark 3.21. Although the primary subject of this paper is intra-step interaction between an algorithm and its environment, it is not our intention to prohibit inter-step interaction of the sort described in 22 and mentioned in 2.1.1. Inter-step interaction is, however, quite easy to describe; the environment is permitted to make arbitrary changes to the state's dynamic functions between the steps of the algorithm. Thus, a run of an algorithm is a (finite or infinite) sequence of states, starting with an initial state, in which each state but the first is obtained from its predecessor either by an intervention of the environment or by a step of the algorithm. In the intervention case, the successor state has the same base set and static functions as the predecessor, but the dynamic functions can be arbitrarily altered. In the case of an algorithm step, there is a successful final history $\xi$, describing the environment's replies to the algorithm's queries during the step, and if the state before the step was $X$ then the state after the step is $\tau(X, \xi)$. Since what happens in the intervention steps is quite arbitrary, our concern in this paper is to analyze what happens in the algorithmic steps. The notion of run, therefore, occurs only in peripheral remarks like this one, not in the main development.

A referee asked why both intra-step and inter-step interaction are needed. In fact, one could eliminate inter-step interaction, but it would require considerable cooperation from the environment because of the following complications. Note that there is no a priori bound on the number of locations that the environment could update in an inter-step action. So that action cannot be simulated by a small-step algorithm in one step. This problem can be circumvented by having the algorithm ask about these environmental updates only when they are needed for the computation. This means that the environment must "remember" these updates until they are needed; furthermore, it must keep track of whether these updates are overwritten by the algorithm's own updates.

3.4. Isomorphism. As in previous work, starting with [23], we require that the information relevant to the algorithm's computation that is given by the state must be explicitly given by the structure of the state, not implicitly given by the particular nature of its elements. Formally, this means that the computation must be invariant under isomorphisms.

Any isomorphism $i: X \cong Y$ between states can be extended in an obvious, canonical way to act on queries, answer functions, histories, locations, updates, etc. We use the same symbol $i$ for all these extensions.

Isomorphism Postulate Suppose $X$ is a state and $i: X \cong Y$ is an isomorphism of $\Upsilon$-structures. Then:

- $Y$ is a state, initial if $X$ is.

- $i$ preserves causality, that is, if $\xi \vdash_{X} q$ then $i(\xi) \vdash_{Y} i(q)$.

- $i$ preserves finality, success, and failure, that is, $i\left(\mathcal{F}_{X}^{+}\right)=\mathcal{F}_{Y}^{+}$and $i\left(\mathcal{F}_{X}^{-}\right)=\mathcal{F}_{Y}^{-}$.

- $i$ preserves updates, that is, $i\left(\Delta^{+}(X, \xi)\right)=\Delta^{+}(Y, i(\xi))$ for all histories $\xi$ for $X$. 
Convention 3.22. In the last part of this postulate, and throughout this paper, we adopt the convention that an equation between possibly undefined expressions is to be understood as implying that if either side is defined then so is the other.

Remark 3.23. We have required that isomorphisms preserve even the irrelevant parts of the algorithm, like update sets for unattainable or non-final histories. This requirement could be dropped, thereby weakening our postulate, without any damage to our results. Nevertheless, it seems a natural requirement in a general description of algorithms. The intuition behind it is that, even if an algorithm includes irrelevant information, the states should still be abstract; not even the irrelevant information should be able to "see" the particular nature of the elements of the state. For example, if $i: X \rightarrow Y$ is an isomorphism, and $\xi$ is an unattainable (hence irrelevant) history in $\mathcal{F}_{X}$, then our postulate requires $i(\xi)$ (which is equally unattainable and thus irrelevant in $Y$ ) to be in $\mathcal{F}_{Y}$. To allow the contrary would be to allow the algorithm to refer to the difference between $X$ and $Y$, a difference that, because of the isomorphism, involves only the particular elements constituting these states, not their abstract structure.

3.5. Small steps. The final postulate formalizes the requirement that a small-step algorithm can do only a bounded amount of work in any one step. The bound depends only on the algorithm. Work includes assembling queries (from elements of the state and labels) and issuing them, computing what queries to issue, deciding whether the current history suffices to finish the step, deciding whether the computation has failed, and computing updates. Our formalization of this closely follows the corresponding postulate in [7, Section 5]; the use of a set of terms to represent the bounded part of the state that the algorithm looks at goes back to 23 .

\section{Bounded Work Postulate}

- There is a bound, depending only on the algorithm, for the lengths of the tuples in Issued $_{X}(\xi)$, for all states $X$ and final, attainable histories $\xi$.

- There is a bound, depending only on the algorithm, for the cardinality $\left|\operatorname{Issued}_{X}(\xi)\right|$, for all states $X$ and final, attainable histories $\xi$.

- There is a finite set $W$ of $\Upsilon$-terms (possibly involving variables), depending only on the algorithm, with the following property. Suppose $X$ and $X^{\prime}$ are two states and $\xi$ is a history for both of them. Suppose further that each term in $W$ has the same value in $X$ as in $X^{\prime}$ when the variables are given the same values in $\operatorname{Range}(\dot{\xi})$. Then:

- If $\xi \vdash_{X} q$ then $\xi \vdash_{X^{\prime}} q$ (so in particular $q$ is a query for $X^{\prime}$ ).

- If $\xi$ is in $\mathcal{F}_{X}^{+}$or $\mathcal{F}_{X}^{-}$, then it is also in $\mathcal{F}_{X^{\prime}}^{+}$or $\mathcal{F}_{X^{\prime}}^{-}$, respectively.

$-\Delta^{+}(X, \xi)=\Delta^{+}\left(X^{\prime}, \xi\right)$.

This completes our list of postulates, so we are ready to define the class of algorithms to be treated in this paper and [10].

Definition 3.24. An interactive, small-step algorithm is any entity satisfying the States, Interaction, Step, Isomorphism, and Bounded Work Postulates. 
Since these are the only algorithms under consideration in most of this paper, we often omit "interactive, small-step"; on the other hand, when we want to emphasize the difference between these algorithms and the "ordinary algorithms" treated in [7, 8, 9, we may refer to the present class of algorithms as "general, interactive, small-step algorithms".

As we explained near the beginning of this section, Definition 3.24 provides a formal meaning for "(interactive, small-step) algorithm", whereas the preceding discussion, leading up to the postulates, was based on an informal notion of (interactive, small-step) algorithm. The definition expresses our belief that the formal notion accurately represents the informal one, i.e., that our postulates adequately describe the intuitive notion.

The remainder of this section is devoted to some terminology and results connected with the Bounded Work Postulate.

Definition 3.25. A set $W$ with the property required in the third part of the Bounded Work Postulate is called a bounded exploration witness for the algorithm. Two pairs $(X, \xi)$ and $\left(X^{\prime}, \xi\right)$, consisting of states $X$ and $X^{\prime}$ and a single $\xi$ that is a history for both, are said to agree on $W$ if, as in the postulate, each term in $W$ has the same value in $X$ as in $X^{\prime}$ when the variables are given the same values in Range $(\dot{\xi})$.

The first two parts of the Bounded Work Postulate assert bounds for final, attainable histories. They imply the corresponding bounds for all attainable histories, thanks to the following lemma.

Lemma 3.26. Let $X$ be a state.

- Any coherent history for $X$ is an initial segment of a complete, coherent history for $X$.

- Any attainable history for $X$ is an initial segment of a final, attainable history for $X$.

Proof. Since $X$ is fixed throughout the proof, we omit it from the notation.

To prove the first assertion, let $\xi$ be a coherent history. According to the definition, its order-type (meaning, strictly speaking, the order-type of the ordering induced by $\leq_{\xi}$ on the equivalence classes under $\equiv_{\xi}$ ) is some ordinal number $\alpha$. We shall inductively define a sequence of coherent histories $\xi_{n}$, starting with $\xi_{0}=\xi$. Here $n$ will range over either the set $\mathbb{N}$ of all natural numbers or the set of natural numbers up to some finite $N$ to be determined during the construction. After $\xi_{n}$ is defined, if it is complete, then stop the construction, i.e., set $N=n$. If $\xi_{n}$ is incomplete, this means that the set $D_{n}=$ $\operatorname{Pending}\left(\xi_{n}\right)=\operatorname{Issued}\left(\xi_{n}\right)-\operatorname{Dom}\left(\dot{\xi}_{n}\right)$ is nonempty. Extend the answer function $\dot{\xi}_{n}$ by adjoining $D_{n}$ to its domain and assigning it arbitrary values (in $X$ ) there. Call the resulting answer function $\dot{\xi}_{n+1}$, and make it into a history $\xi_{n+1}$ by pre-ordering its domain as follows. On $\operatorname{Dom}\left(\dot{\xi}_{n}\right), \leq_{\xi_{n+1}}$ agrees with $\leq_{\xi_{n}}$. All elements of $\operatorname{Dom}\left(\dot{\xi}_{n+1}\right)$ are $\leq_{\xi_{n+1}}$ all elements of $D_{n}$. Elements of $D_{n}$ are $\leq_{\xi_{n+1}}$ only each other. In other words, we extend the ordering of $\operatorname{Dom}\left(\dot{\xi}_{n}\right)$ by adding $D_{n}$ at the end, as a single equivalence class. It is straightforward to check that each $\xi_{n}$ (if defined, i.e., if the sequence hasn't ended before $n$ ) is a coherent history; the order-type of its equivalence classes is $\alpha+n$.

If $\xi_{n}$ is defined for only finitely many $n$, then this is because the last $\xi_{n}$ was complete, and so we have the required result. It remains to consider the case where $\xi_{n}$ is defined for all natural numbers $n$. In this case, let $\zeta$ be the union of all the $\xi_{n}$ 's. (More formally, if we regard functions and orderings as sets of ordered pairs, then $\dot{\zeta}$ is the union of the $\dot{\xi}_{n}$ 's, and the pre-order $\leq_{\zeta}$ is the union of the $\leq_{\xi_{n}}$ 's.) Then $\zeta$ is also coherent. Indeed, if $q \in \operatorname{Dom}(\dot{\zeta})$ then 
there is some $n$ such that $q \in \operatorname{Dom}\left(\dot{\xi}_{n}\right)$. As $\xi_{n}$ is coherent, $q \in \operatorname{Issued}\left(\xi_{n} \uparrow(<q)\right)$. But, since each $\xi_{n}$ is an initial segment of the next, and therefore of $\zeta$, we have $\xi_{n}\lceil(<q)=\zeta \uparrow(<q)$ and so $q \in \operatorname{Issued}(\zeta\lceil(<q)$ ). Furthermore, the order-type of $\zeta$ is $\alpha+\omega$ (where $\omega$ is the order-type of the natural numbers), so it is well-ordered.

To finish the proof of the first part of the lemma, we need only check that $\zeta$ is complete. Suppose, toward a contradiction, that $q \in \operatorname{Pending}(\zeta)$. So there is an initial segment $\eta$ of $\zeta$ such that $\eta \vdash q$. By the Interaction Postulate, $\eta$ is finite, so it is an initial segment of $\xi_{n}$ for some $n$. Then

$$
q \in \operatorname{Issued}\left(\xi_{n}\right) \subseteq \operatorname{Dom}\left(\dot{\xi}_{n+1}\right) \subseteq \operatorname{Dom}(\dot{\zeta})
$$

That contradicts the assumption that $q \in \operatorname{Pending}(\zeta)$, so we have shown that $\zeta$ is complete. Thus, $\xi=\xi_{0}$ is an initial segment of the complete, coherent history $\zeta$, and the first assertion of the lemma is proved.

To prove the second assertion, let $\xi$ be an attainable history. In particular, it is coherent, so, by the first assertion, it is an initial segment of a complete, coherent history $\zeta$. By Part A of the Step Postulate, $\zeta$ has an initial segment $\eta$ that is a final history. If $\zeta$ has several initial segments that are final histories, then let $\eta$ be the shortest of them; thus no proper initial segment of $\eta$ is final. Since both $\xi$ and $\eta$ are initial segments of $\zeta$, one of them is an initial segment of the other. Since $\xi$ is attainable and $\eta$ is final, $\eta$ cannot be a proper initial segment of $\xi$. Therefore, $\xi$ is an initial segment of $\eta$. Furthermore, $\eta$ is coherent, because it is an initial segment of the coherent history $\zeta$. It follows, since no proper initial segment of $\eta$ is final, that $\eta$ is attainable, as desired.

The following corollary extends the first assertion in the Bounded Work Postulate to histories that need not be final.

Corollary 3.27. There is a bound, depending only on the algorithm, for the lengths of the tuples in Issued $_{X}(\xi)$ for all states $X$ and all attainable histories $\xi$.

Proof. The bound on lengths of queries issued by final, attainable histories, given by the Bounded Work Postulate, applies to all attainable histories, because these are, by the lemma, initial segments of final ones.

The next corollary similarly extends the second assertion of the Bounded Work Postulate, and adds some related information.

Corollary 3.28. There is a bound, depending only on the algorithm, for $\left|\operatorname{Issued}_{X}(\xi)\right|$, for all states $X$ and attainable histories $\xi$. The same number also bounds $|D o m(\dot{\xi})|$ for all attainable $\xi$.

Proof. By the lemma, any attainable history $\xi$ is an initial segment of a final, attainable history $\zeta$. Then $\operatorname{Issued}_{X}(\xi) \subseteq \operatorname{Issued}_{X}(\zeta)$. The bound provided by the Bounded Work Postulate for $\left|\operatorname{Issued}_{X}(\zeta)\right|$ thus applies to $\xi$ as well. This proves the first assertion of the corollary, and the second follows because $\xi$, being attainable, is coherent, which implies $\operatorname{Dom}(\dot{\xi}) \subseteq \operatorname{Issued}_{X}(\xi)$. 


\section{Equivalence of Algorithms}

One of our principal aims in this paper and its sequel [10] is to show that every algorithm, in the sense defined above, is behaviorally equivalent, in a strong sense, to an ASM. Of course, this goal presupposes a precise definition of the notion of behavioral equivalence, and we devote the present section to presenting and justifying that definition. As in earlier work on the ASM thesis, beginning in [23] and continuing in [5, 7, 8, 9, the definition of equivalence is intended to express the idea that two algorithms behave the same way in all possible situations. We must, of course, make precise what is meant by "behave the same way" and by "possible situations." Much of what needs to be said here was already said in [7. Section 6] in the more restricted context of ordinary interaction, and we shall refer to some of that discussion in motivating our definition of behavioral equivalence.

Part of the definition of equivalence of algorithms is straightforward. As in previous work, we require equivalent algorithms to have the same states, the same initial states, the same vocabulary, and the same labels. The requirement that they agree as to states and initial states is clearly necessary for any comparison at all between their behaviors, specifically the aspect of behavior given by the progression of states in a run. The requirement that they agree as to vocabulary is actually a consequence of agreement as to states (and the requirement, in the definition of algorithm, that there be at least one state), because any structure determines its vocabulary. The requirement that they agree as to labels ensures that the algorithms have, in any state, the same potential queries; this is needed for any comparison between their behaviors, since issuing queries is observable behavior.

The requirements just discussed say that equivalent algorithms agree as to all the items introduced in the States Postulate. It is tempting to go through the remaining postulates, look for statements of the form "the algorithm determines" such-and-such, and require equivalent algorithms to have the same such-and-such. Unfortunately, this approach, which in [23] would produce the correct notion of equivalence, is too restrictive when applied to interactive algorithms in [7] and the present paper.

The difficulties were already pointed out in [7, Section 6] in connection with the causality relation. The examples given there exhibit the following two sorts of problems. First, a causality relation could have instances $\xi \vdash_{X} q$ whose $\xi$ could never actually occur in the execution of the algorithm, for example because $\operatorname{Dom}(\dot{\xi})$ contains queries that the algorithm would never issue. Second, as in [8, Example 6.4], the $\xi$ in an instance $\xi \vdash_{X} q$ of causality could contain redundant elements, such as a query-reply pair that would have to be present in order for another query in $\operatorname{Dom}(\dot{\xi})$ to be issued. Algorithms whose causality relations differ only in such irrelevant ways should count as equivalent. That is, we should care only about what queries the algorithm issues in response to histories that can actually occur when this algorithm runs.

Similar comments apply to the remaining ingredients of an algorithm, in which histories are used. The notions of final, successful, and failing histories and the update sets should not be required to agree completely when two algorithms are equivalent; it suffices that they agree on those histories that can actually occur.

Remark 4.1. We emphasize again that we are dealing here with a situation where only the algorithm and its environment are involved. When several algorithms interact (and we do not choose to consider each as a part of the environment for the others), the situation would be more complex. Consider, for example, two algorithms that function as components in a larger computation. The first of these components might issue a query whose reply is used 
by the second. In that case, instances $\xi \vdash_{X} q$ of the second component's causality relation can be relevant even if that component would never issue the queries in $\operatorname{Dom}(\dot{\xi})$. Some aspects of this situation will arise in [10] when we discuss the "do in parallel" construct of ASMs; a thorough discussion will be provided in [11].

To formalize the preceding discussion, we must still say something about the notion of a history that can actually appear. We have already introduced a precise version of this notion, namely the notion of an attainable history. This notion, however, depends on the causality relation and the notion of finality given with the algorithm. When we define equivalence of two algorithms, which one's attainability should we use? There are two natural choices: Require the algorithms to agree (as to queries issued, finality, success, failure, and updates) on those histories that are attainable for both algorithms, or require agreement on all histories that are attainable under at least one of the algorithms. (There are also some less natural choices, for example to require agreement on the histories attainable under the first of the two algorithms; this threatens to make equivalence unsymmetric.) Fortunately, these options lead, as we shall prove below, to the same notion of equivalence of algorithms. Having advertised our notion of equivalence as a strong one, we take the apparently stronger of the natural definitions as the official one and then prove its equivalence with the apparently weaker one.

Definition 4.2. Two algorithms are behaviorally equivalent if

- they have the same states (therefore the same vocabulary), the same initial states, and the same labels,

- in each state, they have the same attainable histories,

- for each state $X$ and each attainable history $\xi$, they have the same set $\operatorname{Issued}_{X}(\xi)$,

- for each state, the two algorithms have the same attainable histories in $\mathcal{F}_{X}^{+}$and $\mathcal{F}_{X}^{-}$ (and therefore also in $\mathcal{F}_{X}$ ).

- for each state and each attainable, final, successful history, they have the same update sets.

The following lemma shows that the notion of equivalence is unchanged if we delete the second item in the definition and weaken the subsequent ones to apply only to histories that are attainable for both algorithms.

Lemma 4.3. Suppose two algorithms have the following properties.

- They have the same states (therefore the same vocabulary), the same initial states, and the same labels,

- for each state $X$ and each history $\xi$ that is attainable for both algorithms, they have the same set $\operatorname{Issued}_{X}(\xi)$,

- for each state, any history that is attainable in both algorithms and is in $\mathcal{F}_{X}^{+}$or $\mathcal{F}_{X}^{-}$ for one of the algorithms is also in $\mathcal{F}_{X}^{+}$or $\mathcal{F}_{X}^{-}$, respectively, for the other.

- for each state and each history that is attainable for both and final and successful (for one and therefore both), they have the same update sets.

Then these two algorithms are equivalent.

Proof. Comparing the hypotheses of the lemma with the definition of equivalence, we see that it suffices to prove that, under the hypotheses of the lemma, any history that is attainable in a state $X$ for either algorithm must also be attainable in $X$ for the other. 
Indeed, this would directly establish the second clause of the definition, and it would make the subsequent clauses in the definition equivalent to the corresponding hypotheses in the lemma.

Let $A_{1}$ and $A_{2}$ be two algorithms satisfying the hypotheses of the lemma. Fix a state $X$ for the rest of the proof; we shall suppress explicit mention of $X$ in our notations. To prove that $A_{1}$ and $A_{2}$ have the same attainable histories, we first recall that any attainable history (for either algorithm) has finite domain by Corollary 3.28 and therefore has finite length. We can therefore proceed by induction on the length of histories. Since the empty history vacuously satisfies the definitions of "coherent" and "attainable", we need only verify the induction step.

Consider, therefore, a nonempty history $\xi$ that is attainable for $A_{1}$. Let the last equivalence class in $\operatorname{Dom}(\dot{\xi})$ be $L$ and let $\eta$ be the initial segment of $\xi$ obtained by deleting $L$ from the domain. Then $\eta$ is attainable for $A_{1}$ (because $\xi$ is) and therefore also for $A_{2}$ (by induction hypothesis). Furthermore, the linear order of the equivalence classes of $\xi$, being finite, is certainly a well-ordering. So to complete the proof that $\xi$ is attainable for $A_{2}$, it suffices to verify that it satisfies, with respect to $A_{2}$, the first clause in the definition of coherence (that every $q \in \operatorname{Dom}(\dot{\xi})$ is caused by an initial segment that ends before $q$ ) and that no proper initial segment of it is final.

For the first of these goals, observe that the induction hypothesis gives what we need if $q \in \operatorname{Dom}(\dot{\eta})$, so we need only deal with the case that $q \in L$. In this case, we know that $q \in \operatorname{Issued}^{A_{1}}(\eta)$ because $\xi$ is coherent for $A_{1}$. But since $\eta$ is attainable for both algorithms, the second hypothesis of the lemma applies, and we infer that $q \in \operatorname{Issued}^{A_{2}}(\eta)$, as required.

For the second goal, we already know that $\eta$ is attainable for $A_{2}$ and so none of its proper initial segments can be final. The only proper initial segment of $\xi$ not covered by this observation is $\eta$, so it remains only to show that $\eta$ is not final for $A_{2}$. Since $\eta$ is not final for $A_{1}$ (because $\xi$ is attainable for $A_{1}$ ) and since $\eta$ is attainable for both algorithms, the third hypothesis of the lemma immediately gives the required conclusion.

A referee asked why equivalence of algorithms is not defined in a more usual way, by defining a notion of run and then defining two algorithms being equivalent if they have the same runs. The alternative definition of equivalence is possible but it is not simpler. A run would not be merely a sequence of states as in [23, 5]; it would have to include the queries and replies as well. Note also that our definition of equivalence requires that the two algorithms have the same behavior at every state $X$ whether $X$ is reachable or not. It is thus independent of the set of initial states. To match this, the run-based definition of equivalence should allow runs to start at arbitrary states.

\section{Conclusion}

We have formally defined the class of interactive small-step algorithms (Definition 3.24) by means of postulates that generalize the postulates of [23] and [7], and we have explained why we believe that this definition matches the intuitive notion of interactive small-step algorithm. We have also defined a strong notion of behavioral equivalence for such algorithms. This work provides the essential prerequisites for an analysis of these algorithms in terms of abstract state machines and for the proof of the ASM thesis in thee small-step interactive case. That analysis and proof will be carried out in [10], beginning with the definition of the appropriate class of ASMs. 


\section{REFERENCES}

[1] Alfred Aho, John Hopcroft, and Jeffrey Ullman, The Design and Analysis of Computer Algorithms, Addison-Wesley (1974).

[2] ASM Michigan webpage, http://www.eecs.umich.edu/gasm/ maintained by James K. Huggins.

[3] The AsmL webpage, http://research.microsoft.com/foundations/AsmL/.

[4] Nick Benton, Luca Cardelli and Cédric Fournet, "Modern Concurrency Abstractions for C\#," in Proceedings of the 16th European Conference on Object-Oriented Programming (ECOOP 2002). June 1014, 2002 University of Málaga, Spain, B. Magnusson, ed., Springer-Verlag, Lecture Notes in Computer Science 2374.

[5] Andreas Blass and Yuri Gurevich, "Abstract state machines capture parallel algorithms," ACM Trans. Computational Logic, 4 (2003) 578-651.

[6] Andreas Blass and Yuri Gurevich, "Algorithms: A quest for absolute definitions," Bull. Europ. Assoc. Theoret. Comp. Sci. 81 (2003) 195-225.

[7] Andreas Blass and Yuri Gurevich, "Ordinary Interactive Small-Step Algorithms, I," ACM Trans. Computational Logic 7 (2), 363-419.

[8] Andreas Blass and Yuri Gurevich, "Ordinary Interactive Small-Step Algorithms, II," ACM Trans. Computational Logic, to appear.

[9] Andreas Blass and Yuri Gurevich, "Ordinary Interactive Small-Step Algorithms, III," ACM Trans. Computational Logic, to appear.

[10] Andreas Blass, Yuri Gurevich, Dean Rosenzweig, and Benjamin Rossman, "Interactive small-step algorithms II: Abstract state machines and the characterization theorem" (tentative title), in preparation.

[11] Andreas Blass, Yuri Gurevich, Dean Rosenzweig, and Benjamin Rossman, "Composite interactive algorithms" (tentative title), in preparation.

[12] Andreas Blass, Yuri Gurevich, and Saharon Shelah, "On polynomial time computation over unordered structures," J. Symbolic Logic 67 (2002) 1093-1125.

[13] Egon Börger and Robert Stärk, "Abstract State Machines: A Method for High-Level System Design and Analysis", Springer-Verlag, 2003.

[14] Alonzo Church, "An unsolvable problem of elementary number theory", American Journal of Mathematics 58 (1936), 345-363. Reprinted in [15, 88-107].

[15] Martin Davis, "The Undecidable", Raven Press, 1965.

[16] Andreas Glausch, Varianten des ASM-Theorems, Diploma thesis, Humboldt University, Berlin (2005).

[17] Andreas Glausch and Wolfgang Reisig, "Distributed abstract state machines and their expressive power," preprint (2005).

[18] Dina Q. Goldin, Scott A. Smolka, Paul C. Attie, Elaine L. Sonderegger, "Turing Machines, Transition Systems, and Interaction", Information and Computation 194:2 (2004), 101-128.

[19] Dina Goldin, Scott Smolka and Peter Wegner, editors, Interactive Computation: The New Paradigm, Springer, 2006.

[20] Yuri Gurevich, "A new thesis," Abstract 85T-68-203, Amer. Math. Soc. Abstracts 6 (August, 1985), page 317.

[21] Yuri Gurevich, "Evolving Algebras: An Introductory Tutorial", Bull. EATCS 43 (Feb. 1991), 264284. Reprinted with slight revisions in Current Trends in Theoretical Computer Science: Essays and Tutorials, eds. G. Rozenberg and A. Salomaa World Scientific, 1993, 223-394.

[22] Yuri Gurevich, "Evolving algebra 1993: Lipari guide," in Specification and Validation Methods, E. Börger, ed., Oxford Univ. Press (1995) 9-36.

[23] Yuri Gurevich, "Sequential abstract state machines capture sequential algorithms," ACM Trans. Computational Logic 1 (2000) 77-111.

[24] Yuri Gurevich, "Interactive Algorithms 2005 with Added Appendix", in [19].

[25] Stephen Cole Kleene, Introduction to Metamathematics, Van Nostrand, 1952.

[26] Andrei N. Kolmogorov, "On the concept of algorithm", Uspekhi Mat. Nauk 8:4 (1953), 175-176, Russian. An English translation is found in [35, pages 18-19].

[27] Andrei N. Kolmogorov and Vladimir A. Uspensky, "On the definition of algorithm", Uspekhi Mat. Nauk 13:4 (1958), 3-28, Russian; translated into English in AMS Translations 29 (1963), 217-245.

[28] Nancy L. Lynch, Distributed Algorithms, Morgan Kaufmann Publishers, 1996.

[29] Udi Manber, Introduction to Algorithms: A Creative Approach, Addison-Wesley (1989). 
[30] Yiannis N. Moschovakis, "What is an algorithm?" in B. Engquist and W. Schmid, Editors, Mathematics Unlimited, Springer-Verlag (2001) 919-936.

[31] David S. Platt, Introducing Microsoft .NET, Microsoft Press 2003.

[32] Kay Robbins and Steve Robbins, Unix Systems Programming: Communication, Concurrency and Threads, Prentice Hall Professional Technical Reference, 2003.

[33] Davide Sangiorgi and David Walker, The Pi-calculus: a Theory of Mobile Processes, Cambridge University Press, 2001.

[34] Alan M. Turing, "On computable numbers, with an application to the Entscheidungsproblem", Proceedings of the London Mathematical Society, series 2, vol. 42 (1936-1937), 230265; correction, ibidem, vol. 43, 544-546. Reprinted in [15] 155-222] and available online at http : / www.abelard.org/turpap2/tp2 - ie.asp.

[35] Vladimir A. Uspensky and Alexei L. Semenov, Algorithms: Main Ideas and Applications, Kluwer, 1993. 